LBL-36360

UC-414

\title{
The Physics Program and Status of the SLAC B-Factory and Detector
}

\author{
Christopher Hearty \\ Physics Division \\ Lawrence Berkeley Laboratory \\ University of California \\ Berkeley, California 94720
}

May 1994

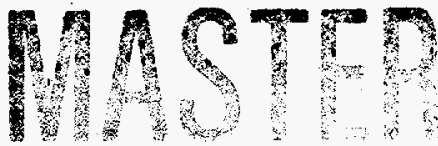

This work was supported by the Director, Office of Energy Research, Office of High Energy and Nuclear Physics, Division of High Energy Physics, of the U.S. Department of Energy under Contract No. DE-AC03$76 \mathrm{SF} 00098$. 


\section{DISCLAIMER}

This report was prepared as an account of work sponsored by an agency of the United States Government. Neither the United States Government nor any agency thereof, nor any of their employees, make any warranty, express or implied, or assumes any legal liability or responsibility for the accuracy, completeness, or usefulness of any information, apparatus, product, or process disclosed, or represents that its use would not infringe privately owned rights. Reference herein to any specific commercial product, process, or service by trade name, trademark, manufacturer, or otherwise does not necessarily constitute or imply its endorsement, recommendation, or favoring by the United States Government or any agency thereof. The views and opinions of authors expressed herein do not necessarily state or reflect those of the United States Government or any agency thereof. 


\section{DISCLAIMER}

Portions of this document may be illegible in electronic image products. Images are produced from the best available original document. 


\title{
THE PHYSICS PROGRAM AND STATUS OF THE SLAC B-FACTORY AND DETECTOR
}

\author{
Christopher Hearty \\ Lawrence Berkeley Laboratory \\ Berkeley, CA 94720
}

\begin{abstract}
The goal of the SLAC B-Factory is to measure CP violation in the decay of B-mesons and to test the standard model description of CP violation. Work is now underway in upgrading the PEP collider for this project, and much of the preliminary detector design has been done. The physics goals and design of PEP-II are briefly discussed, and the current plans for the detector are outlined.
\end{abstract}

\section{INTRODUCTION}

$\mathrm{CP}$ violation has been observed in the laboratory only in the decay of $K^{0}$ mesons. Cosmologically, the matter/antimatter asymmetry in the universe is further evidence that CP is not a conserved quantity. The primary physics goal of the SLAC B-factory is to observe and quantify $C P$ violation in the decay of $B$ mesons and to test the Standard Model description of $\mathrm{CP}$ violation.

\section{PEP-II}

The B-factory - known officially as PEP-II - is an upgrade of the existing PEP collider from a single ring $e^{+} e^{-}$collider to a two ring, asymmetric collider operating at the $\Upsilon(4 S)$ resonance [1]. The $\Upsilon(4 S)$ decays $100 \%$ to $B \bar{B}$ (but sits on a continuum that is approximately three times larger). No conventional construction is required, and approximately $80 \%$ of the existing PEP ring will be reused as the high energy ring.

The two distinctive features of PEP-II are the asymmetric beams, which are needed to increase the typical distance between the decays of the two B-mesons, and its very high design luminosity of $3 \times 10^{33} \mathrm{~cm}^{-2} \mathrm{~s}^{-1}$. The increase in luminosity over PEP or CESR is primarily due to having large number of bunches, giving a time between crossings of $4 \mathrm{~ns}$. The annihilation event rate, however, is only $\sim 14 \mathrm{~Hz}$.

Single beam commissioning will start with the high energy ring in mid-1997 and with the low energy ring approximately six months later. Colliding beam commissioning will start in October 1998.

\section{CP VIOLATION AT THE SLAC B-FACTORY}

The basic CP-violation measurement will be to compare the decay rates for $B^{0}$ and $\overline{B^{0}}$ to a CP-eigenstate fcp [2] [3]. In the case of $B^{0}$ 's coming from the decay of an $\Upsilon(4 S)$, the two $B^{\prime}$ 's are in a coherent state, so that there is one $B^{0}$ and one $\overline{B^{0}}$ until one decays. 
Thus, determining the nature of one $B^{0}$ by its decay products (called tagging) defines the nature of the other one at that instant (defined as $t=0$ ).

$C P$ violation is an interference effect, requiring two amplitudes and two phases. The two amplitudes are due to mixing: the $B$ can decay directly to fcp or it can first mix into a $\bar{B}$, then decay.

In the standard model, the phases are present in the CKM matrix, which governs the strength of the charged weak interaction between quarks. The CKM matrix is unitary, so the rows and columns are orthonormal. One particular relationship (assuming three generations) is:

$$
V_{t d} V_{t b}^{*}+V_{c d} V_{c b}^{*}+V_{u d} V_{u b}^{*}=0
$$

This relationship can be represented as a triangle in the complex plane, called the unitarity triangle for the $B^{0}$ meson (there are five other similar triangles). The angles of this triangle are labeled $\alpha, \beta$ and $\gamma$ : a non-zero angle will, in the standard model, result in $\mathrm{CP}$ violation. The major physics program of the B-factory is to measure at least two angles and two sides of the triangle in order to overconstrain it. Both the angles (CP violation) and the sides (branching ratios) will be measured at the B-Factory [4].

$\mathrm{CP}$ violation will appear as an asymmetry in the decay rate to the final state fcp:

$$
\begin{aligned}
a_{f}(t) & =\frac{\Gamma\left(B^{0}(t) \rightarrow f\right)-\Gamma\left(\bar{B}^{0}(t) \rightarrow f\right)}{\Gamma\left(B^{0}(t) \rightarrow f\right)+\Gamma\left(\bar{B}^{0}(t) \rightarrow f\right)} \\
& \approx-\sin 2 \phi \sin (\Delta M t)
\end{aligned}
$$

where $\phi$ is $\alpha, \beta$ or $\gamma$, and $B^{0}(t)$ is a $B$-meson that was known to be a $B^{0}$ (rather than a $\left.\overline{B^{0}}\right)$ at $t=0$. Note that since $t$ can be positive or negative, the time-integrated asymmetry vanishes. This is a consequence of the B-mesons being produced in a coherent state; it is not true at hadron colliders, for example.

Measurements of CP violation in the $K^{0}$ system and of $V_{u b}$ give a limit of $\sin 2 \beta>$ 0.18 at the $90 \%$ confidence level [2]. Therefore, a significantly higher asymmetry is expected in $B^{0}$ meson decays related to this angle than has been observed in $K^{0}$ mesons, where the asymmetries are in the parts per thousand range.

A CP violation measurement requires tagging one $B$, reconstructing the decay of the other $B$ to $\mathrm{fcp}$, and measuring the time between the decays. Tagging is done by looking for charged kaons, muons or electrons in the final state. The electric charge of these particles is correlated with the charge of the parent b-quark. Cases where this is not true - wrong-sign tags - result both from physics and from detector effects. Wrong-sign tags dilute the statistical significance of the signal. The effective tagging efficiency is $\epsilon_{e f f}=\epsilon_{t a g} \cdot(1-2 w)^{2}$, where $w$ is the fraction of tags that are the wrong sign. The tagging efficiency and wrong-sign fraction are summarized in Table 1 . The wrong sign fraction for kaons is due to physics, whereas both physics and detector effects contribute to the lepton wrong-sign fraction.

The final states fcp are all rare decays. They are summarized in Table 2. There are no $B^{0} \mathrm{CP}$-eigenstates that depend on $\gamma$, so it is unlikely that it will be measured at the Bfactory, at least as part of the initial physics program. The final state $\psi K_{s} \rightarrow l^{+} l^{-} \pi^{+} \pi^{-}$ 


\begin{tabular}{|l|c|c|c|c|}
\hline $\begin{array}{l}\text { Tag } \\
\text { Type }\end{array}$ & $\begin{array}{c}\text { Branching } \\
\text { Fraction }(b) \\
{[\%]}\end{array}$ & $\begin{array}{c}\text { Efficiency } \\
(b \epsilon) \\
{[\%]}\end{array}$ & $\begin{array}{c}\text { Wrong-sign } \\
\text { Fraction }(w) \\
{[\%]}\end{array}$ & $\begin{array}{c}\epsilon(1-2 w)^{2} \\
{[\%]}\end{array}$ \\
\hline \hline Kaons & 37 & 30 & 10 & 19 \\
Muons & 11 & 8.8 & 11.7 & 5.2 \\
Electrons & 11 & 8.5 & 9.9 & 5.5 \\
\hline
\end{tabular}

Table 1: Summary of tagging efficiencies. Overall, $42 \%$ of events are tagged with $w \simeq 10 \%$.

appears the easiest way to measure $\sin 2 \beta$. For an integrated luminosity of $30 \mathrm{fb}^{-1}$ (18 million $B^{0} \overline{B^{0}}$ pairs) we expect to observe approximately 240 tagged events per year, giving an error on $\sin 2 \beta$ of 0.16 . Combining the four modes listed in Table 2 gives an error on $\sin 2 \beta$ of 0.082 .

\begin{tabular}{|l|c|c|c|c|c|c|c|c|}
\hline Mode & $\phi$ & $\begin{array}{c}\text { Br } \\
\left(10^{-4}\right)\end{array}$ & $\begin{array}{c}\text { Recon } \\
\text { Eraction }\end{array}$ & Eff & $\mathrm{d}$ & Events & Bkd & $\delta[\sin 2 \phi]$ \\
\hline \hline$\psi K_{S}$ & $\beta$ & 3.85 & 0.123 & 0.51 & 0.52 & 367 & 0 & 0.13 \\
$\psi K^{* 0}$ & $\beta$ & 12.6 & 0.021 & 0.38 & 0.53 & 150 & 0 & 0.20 \\
$D^{+} D^{-}$ & $\beta$ & 6.0 & 0.067 & 0.26 & 0.53 & 158 & 17 & 0.20 \\
$D^{*+} D^{*-}$ & $\beta$ & 15.0 & 0.105 & 0.12 & 0.52 & 272 & 30 & 0.16 \\
\hline$\pi^{+} \pi^{-}$ & $\alpha$ & 0.2 & 1.000 & 0.35 & 0.54 & 106 & 9 & 0.24 \\
$\rho^{ \pm} \pi^{\mp}$ & $\alpha$ & 0.8 & 1.000 & 0.47 & 0.51 & 567 & 127 & 0.12 \\
\hline
\end{tabular}

Table 2: Summary of $C P$ reach for the $B A B A R$ detector for some major decay modes in a data sample of $30 \mathrm{fb}^{-1}$ accumulated on the $\Upsilon(4 S)$ resonance. "Recon fraction" is the fraction of the mode that goes to a usable final state. " $\mathrm{d}$ " is the dilution factor, the loss of statistical significance due to $B^{0}$ mixing.

\section{THE BABAR DETECTOR}

The preliminary design for the BABAR detector is shown in Fig. 1. It consists of a silicon-strip vertex detector, a low-mass (Helium-based gas) drift chamber, a dedicated particle identification system, a Thallium-doped CsI calorimeter, a $1.5 \mathrm{~T}$ solenoid, and an instrumented-flux return, which serves as both a crude hadronic calorimeter and a muon identification system.

The primary goals of the detector are:

- To observe the decays of $B^{0}$ mesons to a wide range of exclusive final states with high efficiency and low background;

- To tag the flavor of the other B meson in the event with high efficiency and accuracy;

- To measure the relative decay time of the two B mesons. 


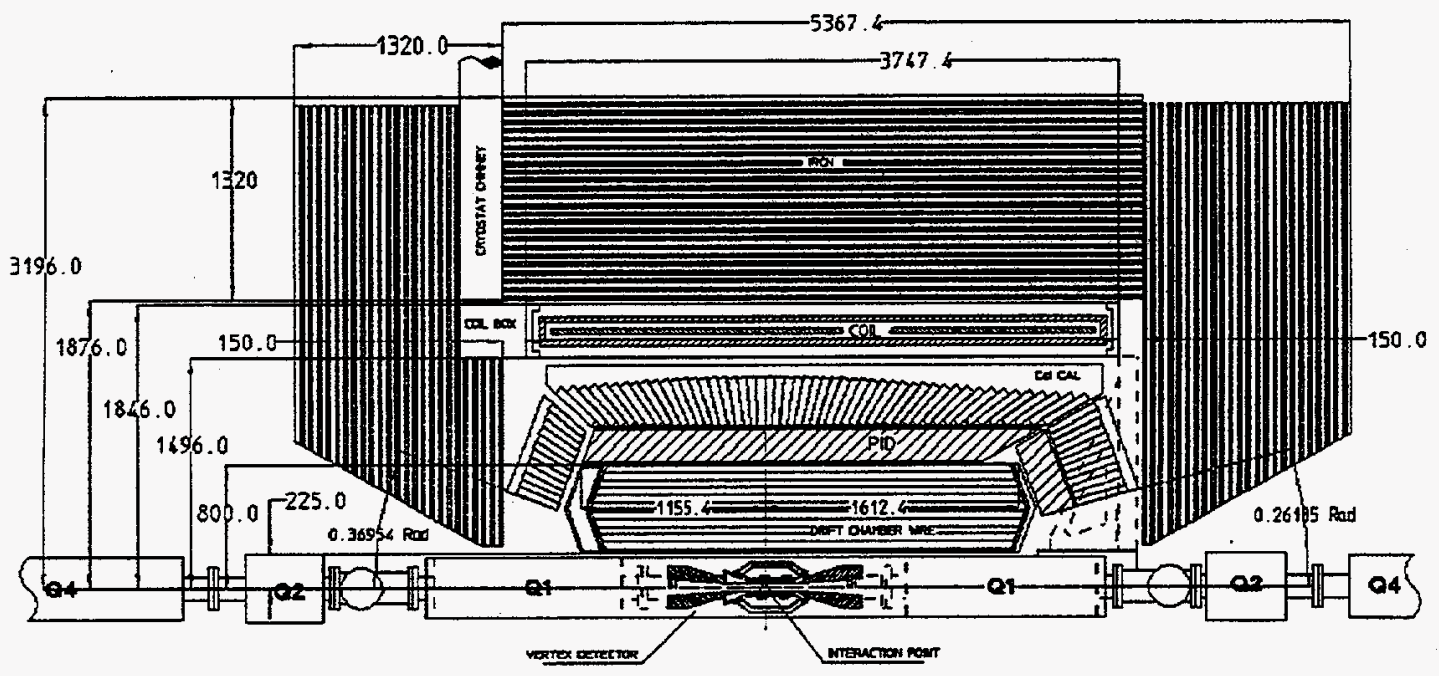

Figure 1: The baseline design of the $B A B A R$ detector as presented in the Letter of Intent. Dimensions in mm.

Because of the boost of the center of mass, good coverage is required in the forward direction. The efficiency for the final state $e^{+} e^{-} \pi^{+} \pi^{-}$is $75 \%$ if the detector has full efficiency to the $300 \mathrm{mr}$ limit imposed by collider components.

Minimal mass is needed to reduce multiple scattering of charged particles and the conversion of photons. At 90 degrees, there is $4 \% X_{0}$ in the vertex detector and beam pipe, $0.5 \% X_{0}$ in a support tube that connects the machine components on either side of the interaction point, $1 \% X_{0}$ in the drift chamber, and $15 \% X_{0}$ in the particle identification system. The support tube reduces the momentum resolution but significantly simplifies the alignment of the machine components.

With this material budget, the 40 layer, $80 \mathrm{~cm}$ outer radius drift chamber achieves a momentum resolution of $\sigma_{p_{t}} / p_{t} \simeq 0.3 \%,\left(p_{t} \leq 1 \mathrm{GeV}\right)$. The calorimeter photon energy resolution goal is $2.5 \%$ at $100 \mathrm{MeV}$ and $1.5 \%$ at $1 \mathrm{GeV}$. In the barrel region, it will have an efficiency of $82 \%$ for $\pi^{\circ} \mathrm{s}$, with a mass resolution of $3.2 \mathrm{MeV}$. This anticipated performance is better than that of CLEO-II due to less inactive material and lower electronic noise.

The measurement of the decay time between the B mesons requires resolution in $z$ (along the beam line) per vertex significantly better than the typical distance of $235 \mu$. This is achieved with a double-sided silicon strip device. There are two layers at the inner radius and two at the outer radius, where the extra layer at each location provides redundancy. A fifth intermediate layer assists in pattern recognition (Fig. 2). The typical $B^{0}$ vertex resolution is expected to be $\leq 50 \mu$.

Tagging the flavor of the other $B$ requires the ability to cleanly distinguish kaons, muons and electrons from other particles, particularly from pions, which are $\sim 7$ times more abundant than kaons and $\sim 15$ times more abundant than electrons and muons. A dedicated ID system is needed to identify charged hadrons. The kaons used for tagging 


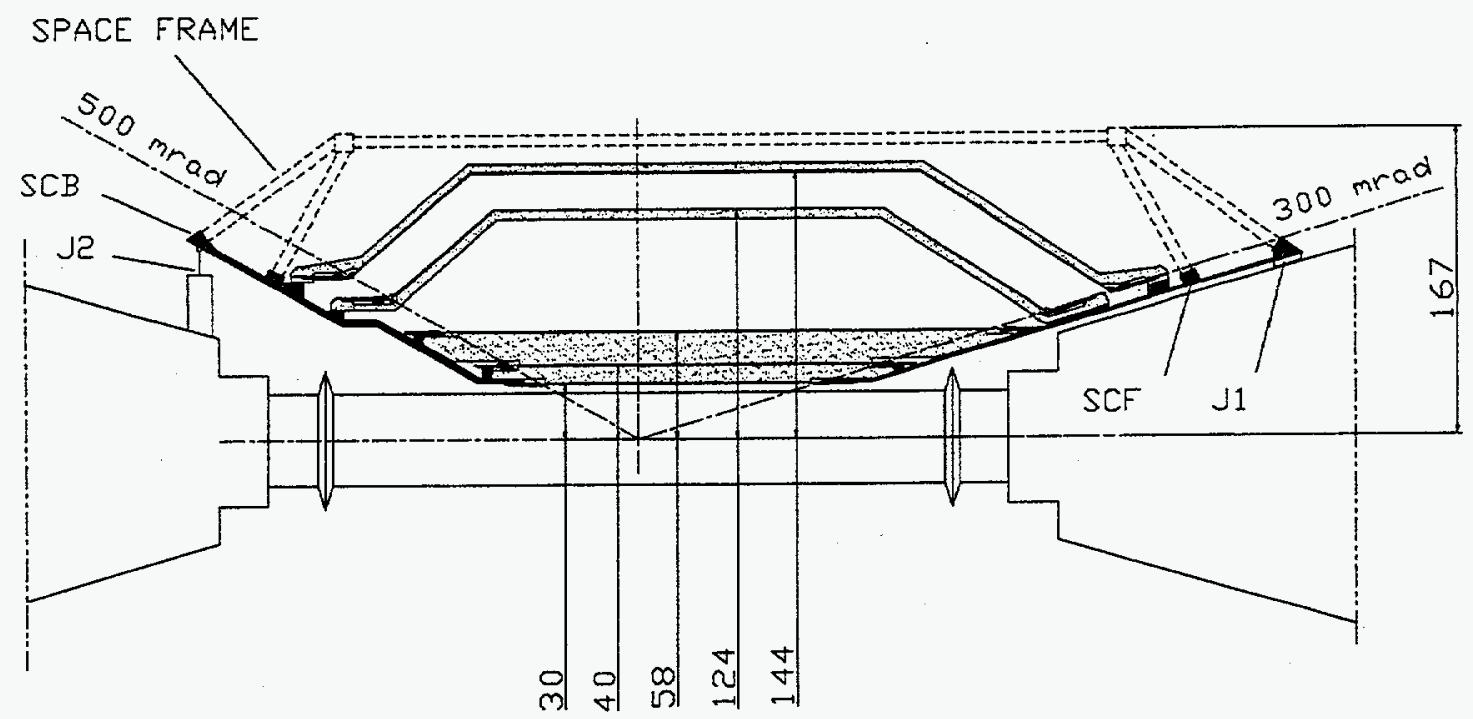

Figure 2: Layout of a vertex detector based on double-sided silicon strip detectors. The radii of five layers in the barrel region are given in $\mathrm{mm}$.

tend to have momenta less than $1.5 \mathrm{GeV}$, while kaons from the interesting exclusive decay $B^{0} \rightarrow \pi K$ can have momenta up to $4.5 \mathrm{GeV}$ in the forward direction. This part of the detector is the most poorly defined in the letter of intent. Three systems are under consideration, each based on the observation of Cerenkov radiation.

The simplest system uses two layers of aerogel to form two thresholds in velocity. The amount of light produced is not large, so the aerogel must be optically clear, and the photosensor - which operates in a $1.5 \mathrm{~T}$ magnetic field - must have good quantum efficiency and good signal-to-noise. Hamamatsu fine-mesh phototubes are being studied as candidate photosensors. It is anticipated that the aerogel could be supplied by JPL, who have produced suitable material for NASA experiments, or Lockheed.

The other two systems are based on reconstructing the Cerenkov ring. The fast RICH has a liquid $C_{6} F_{14}$ radiator separated by a $6 \mathrm{~cm}$ proximity gap from a photosensor used to reconstruct the photon hits. The photocathode is CsI, so the operation is much simpler and the device can operate at higher event rates than TMAE-based devices. It is not yet clear that the fast $\mathrm{RICH}$ will have sufficient light yield to provide $\pi / K$ separation over the full momentum range.

The DIRC is a ring-imaging device that uses Cerenkov light trapped by internal reflection inside a quartz bar (Fig. 3). In the full detector, a layer of bars would be located at the outer radius of the drift chamber, with one end of the bars optically contented to a tank of water located beyond the endcap muon iron. The internally reflected photons travel to the end of the bar, enter the water tank and strike an array of photomultiplier tubes (pmts). The phototube position is used to reconstruct the photon direction. Light traveling along the bar away from the pmts is reflected from a mirror at the far end of the bar. High-momentum particles in the forward direction have a longer path in the quartz and more photoelectrons than lower-momentum particles. A prototype using $47 \mathrm{pmts}$ at a shorter stand-off distance has achieved a resolution of $12 \mathrm{mr}$ per photoelectron on the Cerenkov cone angle. The difference between the cone 


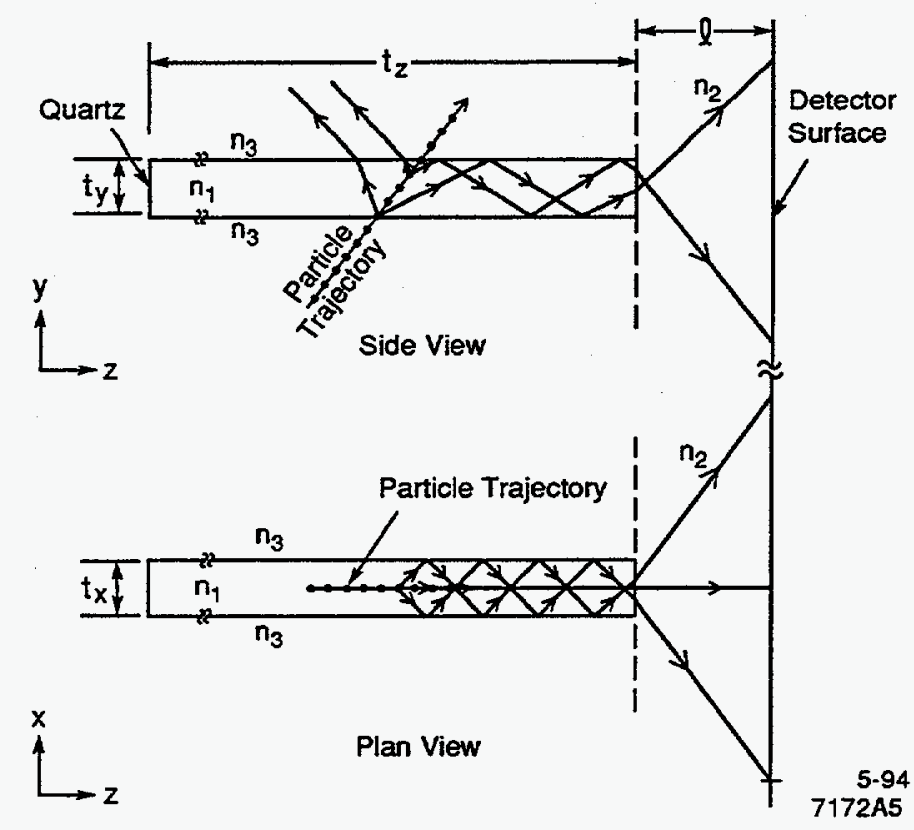

Figure 3: Schematic view of a single radiator bar of a DIRC counter. Representative trajectories of Cerenkov photons are shown by the lines with arrows.

angles of pions and kaons is $5 \mathrm{mr}$ at $4.5 \mathrm{GeV}$. The DIRC makes access to the drift chamber more difficult than the other two systems.

The remaining detector design issues will be resolved for the conceptual design report in late 1994. Procurement of the solenoid - the critical path item - will start in Spring 1995. The goal is to have the detector ready for the commissioning run in October 1998 .

\section{REFERENCES}

1. T. S. Mattison, "Facilities for B-Physics", these Proceedings.

2. The BABAR Collaboration, "Letter of Intent for the Study of CP Violation and Heavy Flavor Physics at PEP-II, SLAC-413, June 1994.

3. D. London, "CP Violation, What's Old, What's New", these Proceedings.

4. The methods for measuring the sides of the unitarity triangle are summarized in: S. Menary, "Observing CP Violation at a Symmetric B-Factory", these Proceedings. 